\title{
The Long Pentraxin PTX3: A Biomarker Spanning From Cardiovascular Disorders to Cancer
}

\author{
Marco Falasca ${ }^{1 *}$, Fabrizia Bonacina ${ }^{2}$, Alberico Luigi Catapano ${ }^{2,3}$ and Giuseppe Danilo Norata ${ }^{1,2,4}$ \\ ${ }^{1}$ Queen Mary University of London, Barts and The London School of Medicine and Dentistry, Blizard Institute, UK \\ ${ }^{2}$ Department of Pharmacological and Biomolecular Sciences, Università degli Studi di Milano, Italy \\ ${ }^{3}$ IRCCS Multimedica, Italy \\ ${ }^{4}$ Center for the Study of Atherosclerosis, Società Italiana Studio Aterosclerosi, Ospedale Bassini, Italy
}

\begin{abstract}
Inflammatory mediators may play key roles in both atherosclerosis and cardiovascular disorders and in cancer development. Pentraxins are a family of proteins characterized by the structural motif pentraxin domain. Long pentraxin 3 (PTX3), a protein that in humans is encoded by the PTX3 gene, is rapidly produced and released by several cell types in response to inflammatory stimuli. This review discusses the evidence supporting the role of PTX3 in predicting primary cardiovascular events and diagnosis and prognosis of cancer.
\end{abstract}

\section{Keywords: PTX3; C-Reactive protein}

\section{Introduction}

Pentraxins are essential components of the humoral arm of innate immunity and represent a family of soluble, multifunctional, pattern recognition proteins. Pentraxins are characterized by a conserved structural motif, the C-terminal pentraxin domain which in the case of Pentraxin 3 (PTX3) is coupled to an unrelated long N-terminal domain. Pentraxins are involved in acute immune-inflammatory responses and are divided into short, such as the $\mathrm{C}$ - reactive protein (CRP), and long pentraxins, such as PTX3 [1].

The pentraxin domain is highly conserved in mammals, in lower vertebrates and in arthropods. The PTX3 gene is highly conserved evolutionary making it possible to address the pathophysiological roles of human PTX3 using genetically modified mice [1]. This is not true for CRP. Furthermore, PTX3 plasma levels increase rapidly in both human and mice peaking at 6-8 hours [2-4] whereas CRP levels increase only in humans in 48 hours during the acute-phase response [1].

Evidence is accumulating indicating that increased PTX3 levels are also associated to cardiovascular disorders [5] and cancer [6,7]. In the last decade, PTX3 has been detected locally at sites of inflammation, such as in the myocardium and in the vasculature under different pathological conditions and in the tumour microenvironment. In addition, increased PTX3 plasma levels have been observed in patients as a consequence of rapid synthesis by various cell types. Data from animal models suggest that this increase might reflect a feedback mechanism involved in dampening an excessive inflammation and not only the consequence of the activation of the immuno-inflammatory system. On the basis of recent data, we will describe in the present review the possible use of PTX3 as a biomarker to improve diagnosis and prognosis of cardiovascular disorders and cancer.

\section{PTX3 Involvement in Biological Pathways}

PTX3 is the prototypic long pentraxin and is rapidly produced by different cell types, such as macrophages, neutrophils, Endothelial Cells (ECs), fibroblasts, vascular Smooth Muscle Cells (SMCs) and epithelial cells in response to several inflammatory cytokines, such as tumour necrosis $\alpha$ and interleukin-1 $\beta$, TRL agonists and microbial components, including lipopolysaccharide (LPS) [8-10]. More recently also anti-inflammatory molecule were shown to modulate PTX3 expression such as IL-10 [11], Glucocorticoid Hormones (GCs) [12], and High-Density Lipoproteins (HDLs), which possess vascular protective properties [13]. HDLs induce PTX3 expression in ECs [14] by activating the phosphoinositide 3-kinase (PI3K)/Akt pathway through G-protein coupled receptors. This is mimicked by sphingosine 1 phosphate (S1P) and others S1P mimetic [14] that are physiologically present in HDL and are responsible for some of the activities linking HDL to the immune inflammatory response [15].

PTX3 possesses multifunctional properties: similarly to short pentraxins, PTX3 recognizes the highly conserved Pathogen-Associated Molecular Patterns (PAMPs) expressed by microorganisms [16] and interacts with C1q, focolin-2 and Mannose-Binding Lectin (MBL) and therefore it modulates the activity of all the three-complement pathways [17], thus playing a crucial role in innate immune responses. More recently, additional unique roles have been discovered connecting PTX3 to inflammation and tissue remodelling. PTX3 competes with leukocyte PSGL-1, thus dampening the recruitment and extravasation of leukocytes mediated by P-selectin and limiting P-selectin-dependent inflammation [18]. Moreover, PTX3 binds with high affinity and selectivity Fibroblast Growth Factor (FGF)-2 [19,20], which in turn results in the inhibition of the interaction of FGF2 with tyrosine-kinase receptors (FGFRs) and Heparin Sulphate Proteoglycans (HSPGs) on the surface of ECs and SMCs [21]. The FGF/FGFR system plays a crucial role not only in FGF2-dependent ECs proliferation [22] but also in SMCs proliferation, migration and survival in vitro [23-26]. These findings suggest that PTX3 is critically involved in both angiogenesis and restenosis, two typical phenomena associated to cancer and cardiovascular diseases.

*Corresponding authors: Marco Falasca, Blizard Institute, Queen Mary University
of London, 4 Newark Street, E1 2AT London, UK, Tel:+44 20 78828243; Fax: +44 20 78822186; E-mail: m.falasca@qmul.ac.uk

Giuseppe Danilo Norata, Department of Pharmacological and Biomolecular Sciences, University of Milan, Via Balzaretti 9, 20133, Milan, Italy, Tel: +39 02 50318313; Fax: +39 02 50318386; E-mail: danilo.norata@unimi.it

Received July 26, 2013; Accepted August 16, 2013; Published August 19, 2013

Citation: Falasca M, Bonacina F, Catapano AL, Norata GD (2013) The Long Pentraxin PTX3: A Biomarker Spanning From Cardiovascular Disorders to Cancer. J Mol Biomark Diagn 4: 146. doi:10.4172/2155-9929.1000146

Copyright: (c) 2013 Falasca M, et al. This is an open-access article distributed under the terms of the Creative Commons Attribution License, which permits unrestricted use, distribution, and reproduction in any medium, provided the original author and source are credited 


\section{PTX3 as a Cardiovascular Biomarker}

While PTX3 deficiency is associated with increased inflammation, cardiac damage [4] and atherosclerosis [27], PTX3 overexpression reduces carotid restenosis after angioplasty $[19,20]$, thus suggesting a protective effect of PTX3 potentially associated with the tuning of inflammation in the context of cardiovascular disorders. Despite this, the role of PTX3 in human still remains controversial: increased plasma levels under diseased conditions might indicate that PTX3 is either a bystander of the inflammatory response or a further attempt to protection of our body. Indeed PTX3 plasma levels peak within 7.5 hours after Myocardial Infarction (MI), as compared to CRP which peaks around 50 hours [3]. In MI patients, PTX3 but not CRP independently predicted 3 months mortality after adjustment for major risk factors and other acute phase proteins [28]. Two large independent clinical trials (CORONA and GISSI-HF), showed that PTX3 was consistently associated with the outcomes in patients with heart failure and with a higher risk of all-cause cardiovascular mortality [29].

\section{PTX3 as a Cancer Biomarker}

Increased levels of PTX3 have been associated with cancer. PTX3 was identified by proteomic approaches as an important candidate biomarker for prostate cancer [6], lung cancer [7], liposarcomas [30] and more recently pancreatic carcinoma [31].

The role of PTX3 in cancer progression remains unknown. Molecular studies indicated that PTX3 exerts an anti-tumoral activity in prostate cancer by inhibiting FGF2/FGF8b-dependent angiogenesis $[32,33]$, and through autocrine and paracrine activities on cancer cells that may contribute to tumour progression [34]. The FGF-binding domain was identified in the PTX $3 \mathrm{~N}$-terminus by monoclonal antibodies and surface plasmon resonance analysis [20], while TSG6, the secreted product of tumour necrosis factor-stimulated gene, was shown to revert the inhibitory effects exerted by PTX3 on FGF2dependent angiogenesis through the interaction with the N-terminal domain of PTX3 [35]. Indeed, overexpression of the PTX3 N-terminus strongly inhibits the tumorigenic activity of TRAMP (Transgenic Adenocarcinoma Mouse Prostate)-C2 transfectants, whereas overexpression of the C-terminus has only a minor effect on tumour growth [32]. Although, PTX3 expression appears to be significantly down-regulated in various human prostate cancer datasets [36], a recent paper by Kondo et al. [31] found a strong correlation between pentraxin family member expression and prognosis of pancreatic carcinoma. The authors propose that pentraxin family members, especially PTX3, may be used as promising biomarkers in the prognosis of pancreatic carcinoma patients.

Given the strong correlation between impaired immune system activity and cancer, the possibility that increased PTX3 plasma levels are correlated to the immune-inflammatory response during cancer, similar to what reported in cardiovascular disorders [37], cannot be excluded.

Interestingly, in contrast with data obtained in prostate cancer, recent data suggest that PTX3 promotes tumour progression by preventing the phagocytosis of tumour cells induced by tumourassociated macrophages in nasopharyngeal carcinoma [38]. This suggests that PTX3 may be a key pro-tumorigenic player in the tumour microenvironment.

Several studies suggest that PTX3 is a potential cancer biomarker. By using proteomic approach five candidate biomarkers were found elevated in serum of lung cancer patients [39]. PTX3 is among these candidates and it was also identified as a candidate prostate [6] and breast [40] cancer biomarker by using a similar approach. Subsequent work has validated PTX3, but not other candidates, in a large cohort of lung carcinoma patients and controls [3]. Indeed, PTX3 serum elevation occurred in $50 \%$ of patients and correlated with the stage disease. PTX3 elevations in serum of lung cancer patients are associated with inflammation and cancer cell apoptosis around the tumour microenvironment.

Recently, PTX3 has been found to be secreted by several pancreatic carcinoma cell lines [13]. In addition, secreted PTX3 has been shown to promote cell migration in a concentration-dependent manner. Evaluation of clinical blood samples for pancreatic carcinoma patients revealed a strong correlation between blood PTX3 level and prognosis of the disease. Furthermore, the examination of the relationship between PTX3 and other pro-inflammatory mediators expression indicated that PTX3 is positively correlated with levels of CRP, interleukin- 6 and macrophage-inhibitory factor. In a more recent study, the expression of PTX3 in gliomas was assessed by immunohistochemistry [41]. PTX3 expression differed across low and high-grade tumours based on histopathological diagnosis and clinical severity, positively correlating with tumour grade and severity.

\section{Conclusions}

PTX3 is an emerging biomarker with application in several pathological conditions including cardiovascular disorders and cancer. Nevertheless, more evidence is required to validate PTX3 as a biomarker with clinical utility either alone or as a member of a biomarker panel. Although the accumulating data pave the road for investigating the relevance of PTX3 as prognostic marker in different cancer conditions and in larger cohorts, several open questions remain: Are PTX3 plasma levels affected by cancer treatment? Are these changes related to the prognosis? Does PTX3 play a protective role in cancer similar to what reported in vitro and in animal studies for cardiovascular diseases? To what extent the ability of PTX3 to dampen FGF response contribute to limit cancer progression?

Answers to these questions and future studies on PTX3 expression in tissues and cells critically involved in both cardiovascular diseases and cancer, such as macrophages, will contribute to improve our understandings on the role of PTX3.

\section{Acknowledgements}

MF is supported by Pancreatic Cancer Research Fund and Prostate Cancer UK.ALC is supported by PUR 2009 University of Milan and by Fondazione Cariplo (2009-2582). GDN is supported by Fondazione Cariplo (2010-0768).

\section{References}

1. Garlanda C, Bottazzi B, Bastone A, Mantovani A (2005) Pentraxins at the crossroads between innate immunity, inflammation, matrix deposition, and female fertility. Annu Rev Immunol 23: 337-366.

2. Mantovani A, Garlanda C, Doni A, Bottazzi B (2008) Pentraxins in innate immunity: from C-reactive protein to the long pentraxin PTX3. J Clin Immunol 28: 1-13.

3. Peri G, Introna M, Corradi D, lacuitti G, Signorini S, et al. (2000) PTX3, A prototypical long pentraxin, is an early indicator of acute myocardial infarction in humans. Circulation 102: 636-641.

4. Salio M, Chimenti S, De Angelis N, Molla F, Maina V, et al. (2008) Cardioprotective function of the long pentraxin PTX3 in acute myocardial infarction. Circulation 117: 1055-1064.

5. Bonacina F, Baragetti A, Catapano AL, Norata GD (2013) Long pentraxin 3 : experimental and clinical relevance in cardiovascular diseases. Mediators Inflamm 2013: 725102 
Citation: Falasca M, Bonacina F, Catapano AL, Norata GD (2013) The Long Pentraxin PTX3: A Biomarker Spanning From Cardiovascular Disorders to Cancer. J Mol Biomark Diagn 4: 146. doi:10.4172/2155-9929.1000146

6. Sardana G, Jung K, Stephan C, Diamandis EP (2008) Proteomic analysis of conditioned media from the PC3, LNCaP, and 22Rv1 prostate cancer cell lines: discovery and validation of candidate prostate cancer biomarkers. J Proteome Res 7: 3329-3338.

7. Diamandis EP, Goodglick L, Planque C, Thornquist MD (2011) Pentraxin-3 is a novel biomarker of lung carcinoma. Clin Cancer Res 17: 2395-2399.

8. Breviario F, d'Aniello EM, Golay J, Peri G, Bottazzi B, et al. (1992) Interleukin-1inducible genes in endothelial cells. Cloning of a new gene related to $\mathrm{C}$-reactive protein and serum amyloid P component. J Biol Chem 267: 22190-22197.

9. Lee GW, Lee TH, Vilcek J (1993) TSG-14, a tumor necrosis factor- and IL1-inducible protein, is a novel member of the pentaxin family of acute phase proteins. J Immunol 150: 1804-1812.

10. Alles VV, Bottazzi B, Peri G, Golay J, Introna M, et al. (1994) Inducible expression of PTX3, a new member of the pentraxin family, in human mononuclear phagocytes. Blood 84: 3483-3493.

11. Doni A, Michela M, Bottazzi B, Peri G, Valentino S, et al. (2006) Regulation of PTX3, a key component of humoral innate immunity in human dendritic cells: stimulation by IL-10 and inhibition by IFN-gamma. J Leukoc Biol 79: 797-802.

12. Doni A, Mantovani G, Porta C, Tuckermann J, Reichardt HM, et al. (2008) Cellspecific regulation of PTX3 by glucocorticoid hormones in hematopoietic and nonhematopoietic cells. J Biol Chem 283: 29983-29992.

13. Norata GD, Pirillo A, Catapano AL (2011) HDLs, immunity, and atherosclerosis. Curr Opin Lipidol 22: 410-416.

14. Norata GD, Marchesi P, Pirillo A, Uboldi P, Chiesa G, et al. (2008) Long pentraxin 3 , a key component of innate immunity, is modulated by high-density lipoproteins in endothelial cells. Arterioscler Thromb Vasc Biol 28: 925-931.

15. Norata GD, Pirillo A, Ammirati E, Catapano AL (2012) Emerging role of high density lipoproteins as a player in the immune system. Atherosclerosis 220 : $11-21$

16. Iwasaki A and Medzhitov R (2010) Regulation of adaptive immunity by the innate immune system. Science 327: 291-295.

17. Bottazzi B, Vouret-Craviari V, Bastone A, De Gioia L, Matteucci C, et al. (1997) Multimer formation and ligand recognition by the long pentraxin PTX3. Similarities and differences with the short pentraxins C-reactive protein and serum amyloid $\mathrm{P}$ component. J Biol Chem 272: 32817-32823.

18. Deban L, Russo RC, Sironi M, Moalli F, Scanziani M, et al. (2010) Regulation of leukocyte recruitment by the long pentraxin PTX3. Nat Immunol 11: 328-334.

19. Camozzi M, Zacchigna S, Rusnati M, Coltrini D, Ramirez-Correa G, et al (2005) Pentraxin 3 inhibits fibroblast growth factor 2-dependent activation of smooth muscle cells in vitro and neointima formation in vivo. Arterioscler Thromb Vasc Biol 25: 1837-1842.

20. Camozzi M, Rusnati M, Bugatti A, Bottazzi B, Mantovani A, et al. (2006) Identification of an antiangiogenic FGF2-binding site in the $\mathrm{N}$ terminus of the soluble pattern recognition receptor PTX3. J Biol Chem 281: 22605-22613.

21. Presta M, Camozzi M, Salvatori G, Rusnati M (2007) Role of the soluble pattern recognition receptor PTX3 in vascular biology. J Cell Mol Med 11: 723-738.

22. Klint $P$ and Claesson-Welsh $L$ (1999) Signal transduction by fibroblast growth factor receptors. Front Biosci 4: D165-177.

23. Koyama H, Reidy MA (1998) Expression of extracellular matrix proteins accompanies lesion growth in a model of intimal reinjury. Circ Res 82: 988-995.

24. Jackson CL, Reidy MA (1993) Basic fibroblast growth factor: its role in the control of smooth muscle cell migration. Am J Pathol 143: 1024-1031.

25. Fox JC, Shanley JR (1996) Antisense inhibition of basic fibroblast growth factor induces apoptosis in vascular smooth muscle cells. J Biol Chem 271: 1257812584.

26. Miyamoto T, Leconte I, Swain JL, Fox JC (1998) Autocrine FGF signaling is required for vascular smooth muscle cell survival in vitro. J Cell Physiol 177 58-67.
27. Norata GD, Marchesi P, Pulakazhi Venu VK, Pasqualini F, Anselmo A, et al. (2009) Deficiency of the long pentraxin PTX3 promotes vascular inflammation and atherosclerosis. Circulation 120: 699-708.

28. Latini R, Maggioni AP, Peri G, Gonzini L, Lucci D, et al. (2004) Prognostic significance of the long pentraxin PTX3 in acute myocardial infarction. Circulation 110: 2349-2354

29. Latini R, Gullestad L, Masson S, Nymo SH, Ueland T, et al. (2012) Pentraxin-3 in chronic heart failure: the CORONA and GISSI-HF trials. Eur J Heart Fail 14 992-999.

30. Willeke F, Assad A, Findeisen P, Schromm E, Grobholz R, et al. (2006) Overexpression of a member of the pentraxin family (PTX3) in human soft tissue liposarcoma. Eur J Cancer 42: 2639-2646.

31. Kondo S, Ueno H, Hosoi H, Hashimoto J, Morizane C, et al. (2013) Clinica impact of pentraxin family expression on prognosis of pancreatic carcinoma. $\mathrm{Br}$ J Cancer 109: 739-746.

32. Ronca R, Alessi P, Coltrini D, Di Salle E, Giacomini A, et al. (2013) Long pentraxin-3 as an epithelial-stromal fibroblast growth factor-targeting inhibitor in prostate cancer. J Pathol 230: 228-238.

33. Rusnati M, Camozzi M, Moroni E, Bottazzi B, Peri G, et al. (2004) Selective recognition of fibroblast growth factor-2 by the long pentraxin PTX3 inhibits angiogenesis. Blood 104: 92-99.

34. Acevedo VD, Ittmann M, Spencer DM (2009) Paths of FGFR-driven tumorigenesis. Cell Cycle 8: 580-588.

35. Leali D, Inforzato A, Ronca R, Bianchi R, Belleri M, et al. (2012) Long pentraxin 3/tumor necrosis factor-stimulated gene-6 interaction: a biological rheostat for fibroblast growth factor 2-mediated angiogenesis. Arterioscler Thromb Vasc Biol 32: 696-703.

36. Rhodes DR, Yu J, Shanker K, Deshpande N, Varambally R, et al. (2004) ONCOMINE: a cancer microarray database and integrated data-mining platform. Neoplasia 6: 1-6.

37. Norata GD, Garlanda C, Catapano AL (2010) The long pentraxin PTX3: a modulator of the immunoinflammatory response in atherosclerosis and cardiovascular diseases. Trends Cardiovasc Med 20: 35-40.

38. Hsiao YW, Li CF, Chi JY, Tseng JT, Chang Y, et al. (2013) CCAAT/Enhancer Binding Protein $d$ in Macrophages Contributes to Immunosuppression and Inhibits Phagocytosis in Nasopharyngeal Carcinoma. Sci Signal 6: ra59.

39. Planque C, Kulasingam V, Smith CR, Reckamp K, Goodglick L, et al. (2009) Identification of five candidate lung cancer biomarkers by proteomics analysis of conditioned media of four lung cancer cell lines. Mol Cell Proteomics 8: 2746 2758

40. Pavlou MP, Dimitromanolakis A, Diamandis EP (2013) Coupling proteomics and transcriptomics in the quest of subtype-specific proteins in breast cancer. Proteomics 13: 1083-1095.

41. Locatelli M, Ferrero S, Martinelli Boneschi F, Boiocchi L, Zavanone M, et al. (2013) The long pentraxin PTX3 as a correlate of cancer-related inflammation and prognosis of malignancy in gliomas. J Neuroimmunol 260: 99-106. 